\title{
Micromechanical model of the single fiber fragmentation test
}

\section{Sørensen, Bent F.}

Published in:

Mechanics of Materials

Link to article, DOI:

10.1016/j.mechmat.2016.10.002

Publication date:

2017

Document Version

Peer reviewed version

Link back to DTU Orbit

\section{Citation (APA):}

Sørensen, B. F. (2017). Micromechanical model of the single fiber fragmentation test. Mechanics of Materials, 104, 38-48. https://doi.org/10.1016/j.mechmat.2016.10.002

\section{General rights}

Copyright and moral rights for the publications made accessible in the public portal are retained by the authors and/or other copyright owners and it is a condition of accessing publications that users recognise and abide by the legal requirements associated with these rights.

- Users may download and print one copy of any publication from the public portal for the purpose of private study or research.

- You may not further distribute the material or use it for any profit-making activity or commercial gain

- You may freely distribute the URL identifying the publication in the public portal

If you believe that this document breaches copyright please contact us providing details, and we will remove access to the work immediately and investigate your claim 


\title{
Micromechanical model of the single fiber
}

\section{fragmentation test}

\author{
Bent F. Sørensen
}

Section of Composites and Materials Mechanics, DTU Wind Energy, Ris $\emptyset$ Campus, The Technical University of Denmark, DK-4000 Roskilde, Denmark e-mail: bsqr@dtu.dk

Highlights:

- New analytical model for analysis of single fragmentation tests

- The model separates friction shear stress from interfacial fracture energy

- Practical procedure is proposed for experimental determination of parameters

A shear-lag model is developed for the analysis of single fiber fragmentation tests for the characterization of the mechanical properties of the fiber/matrix interface in composite materials. The model utilizes the relation for the loss in potential energy of Budiansky, Hutchinson and Evans. The model characterizes the interface in terms of an interfacial fracture energy and a frictional sliding shear stress. Results are obtained in closed analytical form. An experimental approach is proposed for the determination of the interfacial fracture energy and the frictional shear stress from simultaneously obtained data for the applied strain, the opening of a broken fiber and the associated debond length. The residual stresses are obtained as a part of the approach and enables the determination of in-situ fiber strength. 


\section{Keywords: large-scale sliding, fracture mechanics, debonding, residual stresses, interfacial frictional sliding shear stress}

\section{Background}

It is well-recognized that the overall mechanical properties of composite materials, such as strength, toughness and delamination resistance, depend strongly on the mechanical properties of the fiber/matrix interface (Curtin, 1991; Hutchinson and Jensen, 1990, Feih et al., 2005; Sørensen et al., 2008). The idea to characterize the fiber/matrix interface in terms of a critical shear stress determined from the saturated lengths of broken fiber fragments originated from the classical work by Kelly and Tyson (1965). More detailed stress analysis has shown that the elastic shear stress ahead of a fiber break is highly non-uniform (Graciani et al., 2009). Nevertheless, the single fiber fragmentation test remains widely used to characterize polymer matrix composites due to its simplicity, since breakage of fibers and thus the spacing between fiber breaks can easily be determined by conventional optical microscopy for test specimens consisting of transparent matrix materials (Tripathi and Jones, 1998).

The description of the interface in terms of a single strength value has been challenged. Outwater and Murphy (1969) propose to characterize the mechanics of the fiber/matrix interface in terms of an interfacial fracture energy and a frictional shear stress acting along the debonded surface. They also developed a model to determine the interfacial fracture energy from measurements of the applied stress and the debond length of a broken or cut fiber. Since then, more advanced fracture mechanics model have been developed. Many of these models also include a more accurate description of the stress state, include residual stresses and account for 
Poisson's effects of the fibers and matrix and describe the mechanics of the debonded interface by Coulomb friction. As a result, most of these models appear complicated (Wagner et al., 1995; Wu et al., 2000; Graciani et al., 2009); some models only exists as numerical models so that a parameter study must be conducted for each test series to determine interface parameters; this is obviously not a very efficient approach for the analysis of data from single fiber fragmentation tests. In other cases, additional parameters, such as the residual stresses and/or the friction coefficient (in models using Coulomb friction) must be calculated or determined from independent experiments (Nairn, 2000; Ramirez et al., 2009).

In addition, Varna et al. (1996) noted that the fiber breakage and fiber/matrix debonding are two independent features and that debonding may not always occur immediately after fiber breakage but may require a substantial higher strain.

Kim and Nairn (2002a) provided a detailed description, documented by micrographs, of the evolution of damage in single filament specimens subjected to increasing applied strain. They used polarized light to visualize the shear stress field around a broken fiber. They found that an initial debonding developed during the fiber breakage. The debond length and the fiber break gap were found to increase with increasing applied strain, whereas the fiber gap decreased during unloading. The maximum debond length was 16-17 times the fiber diameter (224 microns). Images showed that some fiber break gaps were several times the fiber diameters (up to 30-50 microns). Such observations clearly show that for these material systems, a model based on interfacial fracture energy and sliding friction provides a better description of the fiber/matrix interface than a model based on a constant shear stress. 
Kim and Nairn (2002a) reported both the "whole debond" length (the average value of all debond length of all locations of fiber break of a specimen) and "instantaneous debond length" (the debond length associated with new fiber breaks, i.e. fiber breaks that occurred between the present and previous load step) as a function of applied strain. For glass/epoxy, Kim and Nairn (2002a) found that the data for "whole debonds" and "instantaneous debonds" were identical for small applied strain (indicating little or no interaction between the fiber breaks, i.e., isolated fiber breaks) but deviate for strains larger than $2.75 \%$, indicating interaction between the locations of fibre break.

Kim and Nairn (2002a) analyzed their experimental data using the analytical model of Nairn (2000), a mathematical model that incorporates Poisson's effects in an approximate way and models the mechanics of the interface in terms of an interfacial fracture energy and Coulomb friction. The model predicts an initial non-linear relationship (progressive increase in debond length) between applied strain and debond length, followed by a linear relationship (isolated fiber breaks) and finally a non-linear relationship (decreasing debond growth rate) for high strains, as fiber breaks interact. In an accompanying study (Kim and Nairn, 2002b), debond data were presented for "instantaneous debonds" only, and only up to the strain value up to $3.0 \%$, i.e. strain values where there were little interaction between fiber breaks. Kim and Nairn (2002b) concluded that to get correct parameter values, the debonding experiments should be combined with other experiments that can measure residual 
stresses and friction. Nevertheless, they identified the interfacial fracture energy to be $120 \mathrm{~J} / \mathrm{m}^{2}$ and the frictional parameter to be 0.01 .

Graciani et al. (2009) developed a numerical model using the boundary-element method (BEM). The interface was modelled in terms of a fracture energy and Coulomb friction and the model included residual stresses. The model predicts that the debond crack tip singularity becomes weaker (i.e. a power less than 1/2) in the presence of friction. The model predicts that the relationship between debond length and applied strain is non-linear (debond length increasing progressing with increasing strain) for debond length smaller than about five fiber radii, and a linear relationship with a finite, constant slope for larger debond lengths. Apparently, the non-linear relationship between strain and debond length (for small debonds length) is due to a variation in the energy release rate with debond length for fixed overall strain; the energy release rate starts very high and decreases rapidly with increasing debond crack length attaining a steady-state value when the debond crack length exceed about five fiber radii.

Graciani et al. (2011) analyzed the experimental data of Kim and Nairn (2002b) using the numerical BEM model. They obtained best agreement with the experimental data with a Coulomb friction coefficient of 1.0 and an interfacial fracture energy of $12 \mathrm{~J} / \mathrm{m}^{2}$ . Recall that Kim and Nairn (2002b) determined the interfacial fracture energy to be $120 \mathrm{~J} / \mathrm{m}^{2}$ for the same data. It is remarkable that analyzing the same data, the two advanced models (that of Nairn (2000) and that of Graciani et al. (2011)) identify parameters that are widely different, despite both incorporate residual stresses, Coulomb friction and Poisson's effects. There is thus a need for a clearer approach for 
parameter identification. A drawback of the advance models is that they are a bit complicated to use - parameters are not determined in a straight forward manner - and it is not possible - due to model complexity - to see the sensitivity of each parameter on model predictions. The simple shear-lag model that is developed in the present paper enables a clearer parameter identification from experiments data.

The accuracy of analytical shear-lag models can be assessed by comparing their predictions with predictions from more accurate numerical models. Such comparisons were made by Hutchinson and Jensen (1990) who developed closed form analytical shear lag models for fibre debonding and pull-out. The fiber/matrix interface was modelled in terms of an interfacial fracture energy and a constant interfacial frictional shear stress or by Coulomb friction. The model also includes Poisson's effects and residual stresses. They compared results from the analytical model with accurate numerical results. They found that the energy release rate of the numerical model approach that of the analytical model when the debond length is larger than about one fiber radius. They also compared the displacement difference between the lower end of fiber and matrix and found that the analytical model become increasing accurate as the debond length grows. According to Hutchinson and Jensen (1990), the difference should not be of much significance when the debond length is longer than about five times the fiber radius.

The proper way of mechanical characterization of frictional sliding remains an open issue. Mackin et al. (1992) and Liang and Hutchinson (1993) have proposed an interfacial friction sliding law of the form 


$$
\tau_{s}=\tau_{0}-\mu \sigma_{r r} \quad \text { for } \sigma_{r r} \leq 0
$$

where $\tau_{s}$ is the frictional sliding shear stress, $\tau_{0}$ represents friction introduced by roughness, $\mu$ the Coulomb friction coefficient and $\sigma_{r r}$ is the radial normal stress at the debonded fiber/matrix interface. Connell and Zok (1997) found that such a description could represent the sliding behavior of a ceramic fiber composite well. They found that a constant sliding shear stress could described the sliding behavior well at one temperature; the second term in (1) allows the description of different frictional sliding stresses at various other temperatures (via changes in the radial normal stress, $\left.\sigma_{r r}\right)$.

The motivation for the present study is thus to develop an analytical model and an approach for the determination of the interfacial fracture energy and the frictional sliding shear stress from a tensile specimen consisting of a single filament embedded in a matrix specimen subjected to uniaxial tension. More specifically, we wish to develop a practical approach for the determination of the frictional shear stress, $\tau_{s}$, and the interfacial fracture energy, $\mathscr{\varphi}_{c}^{i}$ from experiments, accounting for the residual stresses.

In the present paper, we propose to use data for the broken fiber gap as an additional experimental parameter to measure - building on the conclusion of Kim and Nairn (2002b) that additional experimental data are needed. Moreover, we propose a new 1D model that is simpler that the models of Wu et al. (2000) and Nairn (2000). An advantage of the new, simpler model is that it gives a clearer relationship between interface parameters and measure properties so that it becomes easier to assess the 
effect of interfacial parameters from experimental data. Being a shear-lag model, it only applies for debond lengths larger than about five fiber radii.

Obviously, the hope is that the model enables the determination of interface parameters which can be considers being material properties and thus be used in micromechanical models of composites with much higher fiber volume fraction (typically, 40-60\% in engineering composites). This would enable tests of single filament composites to be used as the primary tool for characterization of mechanical properties of fiber/matrix interface. Investigations of how changes in fiber surface treatments, composite processing conditions alters the mechanical properties of the fiber/matrix interface (e.g., van der Waals or covalent bonds) will then help the development of composite materials with improved properties. This assumption will be discussed in more details in Section 7.

\section{Problem description}

The problem to be analyzed is a single fiber embedded in a tensile test specimen made of the matrix material as shown in Fig. 1. We consider a situation where the applied stress level is $\bar{\sigma}$, the fiber has broken and a debond crack has formed along a part of the fiber/matrix interface. The location of the fiber breakage is assumed to be remote from other fiber breaks. The model consists of a fiber with a fiber radius, $r$, surrounded by a hollow matrix cylinder. The single filament composite is pre-stressed by residual stresses in the fiber and matrix. However, the fiber is stress-free at the location of the fiber breakage $(z=0)$. Due to symmetry of the problem, only half the specimen $(z \geq 0)$ is analyzed, as shown in Fig. 2 . The fiber is debonded a distance $\ell_{d}$ and the opening displacement of the fiber end is $\delta$ so that the total opening 
displacement of the broken fiber (the fiber gap) is $2 \delta$. Both $\ell_{d}$ and $\delta$ are assumed to depend on $\bar{\sigma}$. A constant frictional shear stress, $\tau_{s}$, is assumed to operate along the debonded interface acting in the direction opposite to the sliding direction.

Furthermore, Poisson's effects are ignored. The problem will be analyzed by a onedimensional shear-lag model. This analysis is assumed to be accurate when the debond length and the length of the uncracked parts are a few times longer than the fiber diameter so that the crack tip stress field is fully evolved and a uniform stress field (denoted upstream stresses) exists ahead of the crack tip in the un-cracked part (Hutchinson and Jensen, 1990).
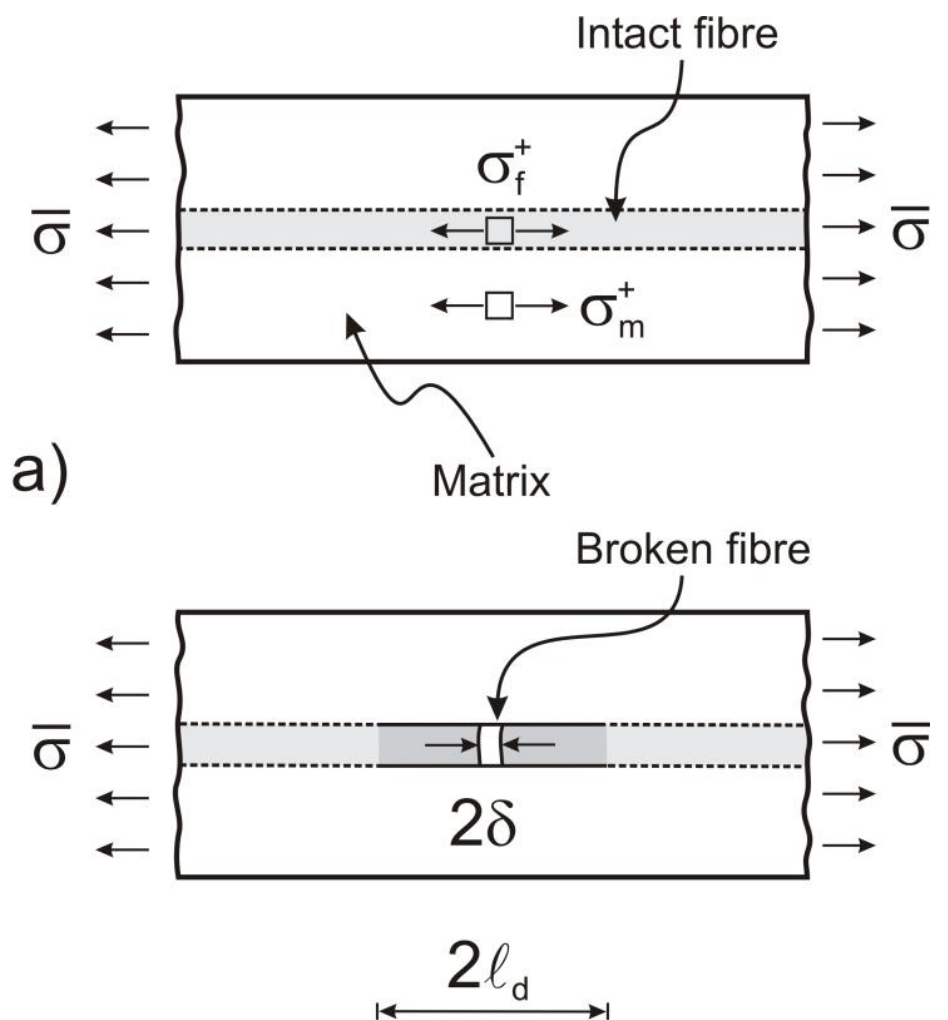

b) Debonded Zone

Fig. 1: Schematics of a single fiber embedded in a matrix test specimen. (a) Definition of stresses in uncracked specimen and (b) details of broken fiber. 
The present model has some similarities with the pull-out models of Hutchinson and Jensen (1990) and Kessler et al., (1999) in that the interface is characterized in terms of a interfacial fracture energy, $\mathscr{G}_{c}^{i}$, and a frictional sliding shear stress, $\tau_{s}$.

The radius of the external surface of the matrix cylinder is denoted $R$. From the geometry we can calculate the fiber volume fraction, $V_{f}$, as

$$
V_{f}=\left(\frac{r}{R}\right)^{2}
$$

The analysis is then valid also for single filament specimens where the radius of the matrix is of same order of magnitude as the fiber radius, sometimes called minicomposites.

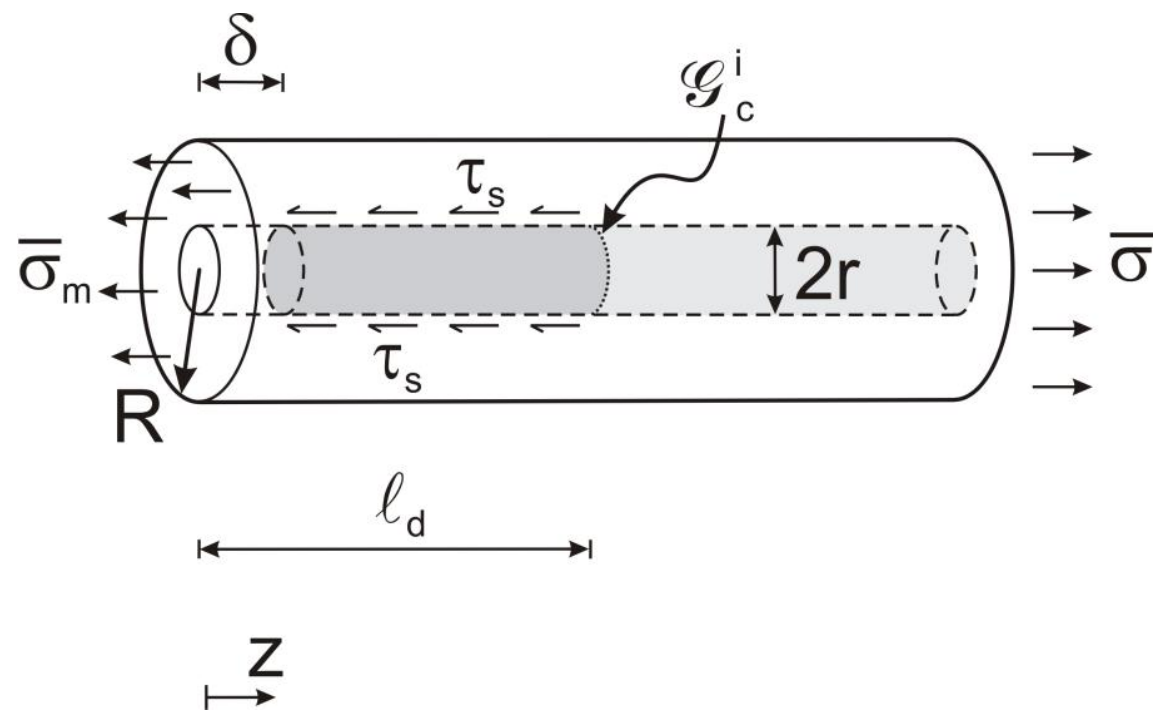

Fig. 2: The analyzed problem: A single fiber embedded in a matrix cylinder undergoing debonding with frictional sliding in the debonded zone.

The strategy to develop the model is as follows. First we determine the stress state in the uncracked part and the stress state in the debonded region. The debond length is initially treated as an unknown but is determined by the use of the relations for the 
potential energy loss of Budiansky, Hutchinson and Evans (1986). Having determined the debond length, we derive equations for the fiber opening displacements, which can subsequently be used in the determination of the unknown interface parameters, $\tau_{s}$ and $\mathscr{G}_{c}^{i}$.

\section{Stress analysis}

\section{$\underline{3.1 \text { Residual stresses }}$}

Residual stresses often develop in composites during processing due to various inelastic phenomena such as cross-linking or phase transition of polymers during curing and thermal expansion coefficient mismatch between the fiber and the matrix, creating residual stresses during cool-down from the processing temperature. In the present study we will make no specific assumption regarding the origin and magnitude of the residual stresses. Instead, the residual stresses will be determined as part of the data analysis. Denote the in-elastic strain (sometimes called the stress-free strain, Eshelby (1957)) of the fiber and matrix by $\varepsilon_{f}^{T}$ and $\varepsilon_{m}^{T}$. We defined a misfit strain,

$$
\Delta \varepsilon^{T}=\varepsilon_{f}^{T}-\varepsilon_{m}^{T}
$$

which we will use henceforward. $\Delta \varepsilon^{T}$ will be positive for most composites with a polymer matrix. Normally, the processing-induced in-elastic strains are negative and numerical largest for the matrix material $\left(\varepsilon_{m}^{T}<\varepsilon_{f}^{T}<0\right)$, but the fiber may be preloaded (e.g. pre-stressed in tension to keep the fiber straight) during the processing (Wagner and Zhou, 1998). As an example, residual stressed induced by thermalexpansion mismatch can be estimated by considering the specimen to be stress-free at temperature $T_{0}$. The fiber is assumed to have a thermal expansion coefficient, $\alpha_{f}$, 
and the matrix has a thermal expansion coefficient, $\alpha_{m}$. Then, after cool-down to temperature $T$, the inelastic strains (defined to be zero at the stress-free state at the processing temperature) are

$$
\varepsilon_{f}^{T}=\alpha_{f}\left(T-T_{0}\right) \quad \varepsilon_{m}^{T}=\alpha_{m}\left(T-T_{0}\right)
$$

so that

$$
\Delta \varepsilon^{T}=\left(\alpha_{f}-\alpha_{m}\right)\left(T-T_{0}\right)
$$

The residual stresses in the fiber and matrix, $\sigma_{f}^{r e s}$ and $\sigma_{m}^{r e s}$, must be in force equilibrium. Assuming that the fiber and matrix materials are linear elastic with a Young's modulus $E_{f}$ and $E_{m}$ respectively, the residual stresses can be written as

$$
\frac{\sigma_{f}^{r e s}}{E_{f}}=-\Delta \varepsilon^{T}\left(1-V_{f}\right) \frac{E_{m}}{E_{c}} \quad \frac{\sigma_{m}^{r e s}}{E_{m}}=\Delta \varepsilon^{T} V_{f} \frac{E_{f}}{E_{c}},
$$

where $E_{c}$ is the Young's modulus of the composite specimen, defined as

$$
E_{c}=V_{f} E_{f}+\left(1-V_{f}\right) E_{m},
$$

with $V_{f}$ being the fiber volume fraction, given by (2).

\subsection{Upstream stresses (stresses far ahead of the debond crack tip)}

We now analyze the stress state after the application of an applied stress, $\bar{\sigma}$. The upstream stress (i.e., the stress acting in the $z$-direction in the uncracked part of the specimen, far ahead of the debond crack tip, $z>>\ell_{d}$ ) in the fiber, denoted $\sigma_{f}^{+}$, and the upstream stress in the matrix, $\sigma_{m}^{+}$, can be found by assuming the same axial strain in the fiber and matrix and by force balance of the specimen in the $z$-direction. The results can be written as 


$$
\frac{\sigma_{f}^{+}}{E_{f}}=-\Delta \varepsilon^{T} \frac{\left(1-V_{f}\right) E_{m}}{E_{c}}+\frac{\bar{\sigma}}{E_{c}} \quad \text { and } \quad \frac{\sigma_{m}^{+}}{E_{m}}=\Delta \varepsilon^{T} V_{f} \frac{E_{f}}{E_{c}}+\frac{\bar{\sigma}}{E_{c}}
$$

\subsection{Downstream stresses (stresses in the sliding zone)}

In the debonded part of the specimen, $0 \leq z \leq \ell_{d}$, the stresses in the fiber and matrix, denoted $\sigma_{f}^{-}$and $\sigma_{m}^{-}$, respectively, vary as a function of $z$-position. These stresses are calculated by the use of a shear-lag analysis. Force balance of the fiber gives the stress in the fiber as

$$
\sigma_{f}^{-}(z)=2 \tau_{s} \frac{z}{r} \quad \text { for } 0 \leq z \leq \ell_{d} .
$$

The debond length $\ell_{d}$ is determined by the use of an energy criterion in the next section. It can be noted that when $\Delta \varepsilon^{T}=0$ and $\mathscr{\vartheta}_{c}^{i}=0$, so that the debond length actually is a frictional slip length, the fiber and matrix stresses become continuous at the crack tip; otherwise there is a "jump" in the stress in fiber and matrix at the position of the debond crack, analogous to the pull-out model of Hutchinson and Jensen (1990).

Force balance of the hollow matrix cylinder gives

$$
\sigma_{m}^{-}(z)=\frac{\bar{\sigma}}{\left(1-V_{f}\right)}-2 \frac{V_{f}}{\left(1-V_{f}\right)} \frac{z}{r} \tau_{s} \quad \text { for } 0 \leq z \leq \ell_{d} .
$$

Note from (9) and (10) that the stresses in the slip zone depend on $z$-position but are otherwise independent of the debond length, $\ell_{d}$.

Now we need to determine the debond length, $\ell_{d}$, as a function of the applied stress, $\bar{\sigma}$. Had there been no friction along the fiber/matrix interface, we could have applied 
linear-elastic fracture mechanics and determined the debond length by requirering that the energy release rate should be equal to the fracture energy of the interface. The presence of large-scale frictional sliding, however, invalidates the use of some linear elastic fracture mechanics approaches, such as the compliance method, for the calculation of the debond crack length. Therefore, the method of Budiansky et al. (1986) will be used for the calculation of the potential energy loss accounting for the frictional energy dissipation. This leads to an equation for the debond length.

\section{Determination of the debond length}

\subsection{The potential energy approach of Budiansky, Hutchinson and Evans}

The potential energy approach of Budiansky et al. (1986) gives the potential energy differences between two states in which cracking and monotonic frictional slip has occurred in a body that initially is pre-stressed by residual stresses. In State I, the body is subjected to surface tractions and some cracking and frictional slippage has occurred. With fixed surface tractions, the body undergoes further debonding and frictional slipping to State II, so that the applied tractions perform work and the frictional shear stress performs further work and thus dissipates energy. Budiansky et al. (1986) used the principle of virtual work to eliminate the work of the applied tractions. The potential energy difference between the two states can then be written as (Budiansky et al., 1986)

$$
\Pi_{I}-\Pi_{I I}=\frac{1}{2} \int_{V}\left(\sigma_{I}-\sigma_{I I}\right): M\left(\sigma_{I}-\sigma_{I I}\right) d V+\xi_{F},
$$

where $\Pi_{I}$ denotes the potential energy of State I while $\Pi_{I I}$ is the potential energy of State II. Moreover, $V$ is the volume of the body, $\sigma_{I}$ is the stress tensor associated with State I, and $\sigma_{I I}$ is the stress tensor associated with State II. Furthermore, $M$ indicates 
an elastic operator (i.e., Hooke's law) and $\xi_{F}$ is the frictional energy dissipation (frictional work) defined as

$$
\xi_{F}=\tau_{s} \int_{S_{F}}|\Delta u| d S
$$

In (12), $|\Delta u|$ denotes the relative frictional slip between the two states and $S_{F}$ is the surface area at which frictional sliding occurs. The slip is assumed to occur monotonically during the transition from State I to State II.

\section{$\underline{4.2 \text { Model development }}$}

For the problem at hand, we identify the two states as shown in Fig. 3. State I constitutes the situation of a debond length $\ell_{d} \gg r$. State II is the situation where the length of the debond crack has increased by a small distance, $\Delta \ell_{d}$. With the tractions at the external boundaries held fixed, the State II stresses, $\sigma_{f}^{-}(z)$ and $\sigma_{m}^{-}(z)$, in the "old" (State I) slip zone $\left(0<z<\ell_{d}\right)$, are exactly the same as for State I; this follows from (9) and (10). The length of the slip zone has increased by $\Delta \ell_{d}$, so that in State II, equations (9) and (10) for $\sigma_{f}^{-}(z)$ and $\sigma_{m}^{-}(z)$ are now valid also for the new (State II) slip zone, $0 \leq z \leq \ell_{d}+\Delta \ell_{d}$. The upstream stresses, $\sigma_{f}^{+}$and $\sigma_{m}^{+}$, remain unchanged for $z>\ell_{d}+\Delta \ell_{d}$. Then, for the transition from State I to State II, the stress state changes only for $\ell_{d}<z<\ell_{d}+\Delta \ell_{d}$. Consequently, (11) becomes

$$
\Pi_{I}-\Pi_{I I}=\frac{1}{2} \int_{\ell_{d}}^{\ell_{d}+\Delta \ell_{d}}\left\{\pi r^{2} \frac{\left(\sigma_{f}^{+}-\sigma_{f}^{-}(z)\right)^{2}}{E_{f}}+\pi\left(R^{2}-r^{2}\right) \frac{\left(\sigma_{m}^{+}-\sigma_{m}^{-}(z)\right)^{2}}{E_{m}}\right\} d z+\xi_{F}
$$

This analysis bypasses the singular crack tip stress fields at the crack tips. Inserting $\sigma_{f}^{+}$and $\sigma_{m}^{+}$from (8) and $\sigma_{f}^{-}(z)$ and $\sigma_{m}^{-}(z)$ from (9) and (10) into (13) gives: 


$$
\begin{aligned}
\Pi_{I}-\Pi_{I I}= & \frac{\pi}{2} r^{2}\left\{\int_{\ell_{d}}^{\ell_{d}+\Delta \ell_{d}} \frac{E_{f}}{E_{c}} \frac{1}{\left(1-V_{f}\right) E_{m}}\left(\left(1-V_{f}\right) E_{m} \Delta \varepsilon^{T}-\bar{\sigma}\right)^{2}+4 \frac{E_{f}}{E_{c}} \frac{1}{\left(1-V_{f}\right) E_{m}} \tau_{s}^{2}\left(\frac{z}{r}\right)^{2}+\right. \\
& \left.4\left(\Delta \varepsilon^{T}-\frac{\bar{\sigma}}{\left(1-V_{f}\right) E_{m}}\right) \tau_{s} \frac{z}{r} d z\right\}+\xi_{F} .
\end{aligned}
$$

Performing the integration gives

$$
\begin{aligned}
\Pi_{I}-\Pi_{I I}= & \frac{\pi}{2} r^{2}\left\{\frac{E_{f}}{E_{c}} \frac{1}{\left(1-V_{f}\right) E_{m}}\left(\left(1-V_{f}\right) E_{m} \Delta \varepsilon^{T}-\bar{\sigma}\right)^{2}\left[\Delta \ell_{d}\right]+4 \frac{E_{c}}{E_{f}} \frac{1}{\left(1-V_{f}\right) E_{m}} \tau_{s}^{2}\left[\frac{\left(\ell_{d}+\Delta \ell_{d}\right)^{3}-\ell_{d}^{3}}{3 r^{2}}\right]\right. \\
& \left.+4\left(\Delta \varepsilon^{T}-\frac{\bar{\sigma}}{\left(1-V_{f}\right) E_{m}}\right) \tau_{s}\left[\frac{\left(\ell_{d}+\Delta \ell_{d}\right)^{2}-\ell_{d}^{2}}{2 r}\right]\right\} \Delta \ell_{d}+\xi_{F} .
\end{aligned}
$$

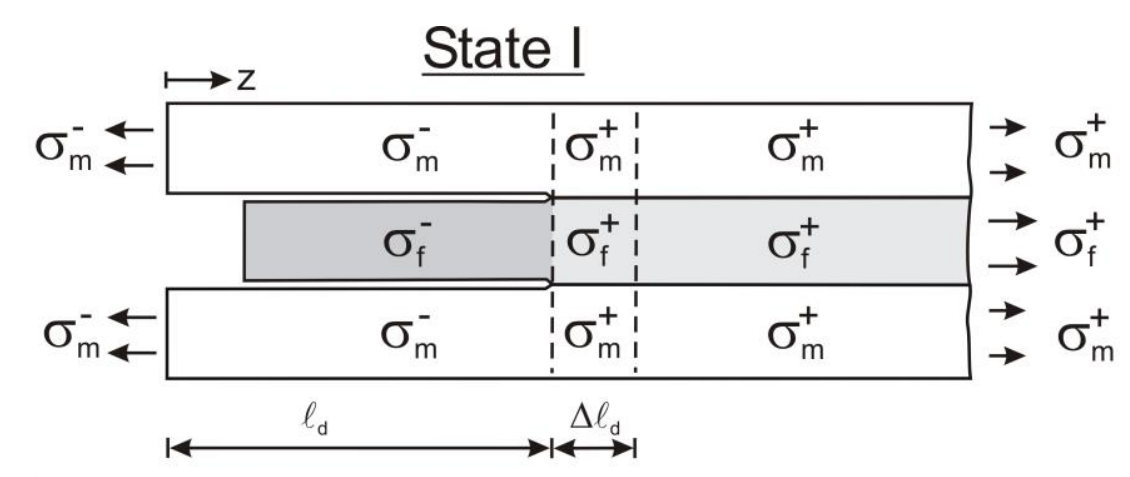

a)

\section{State II}

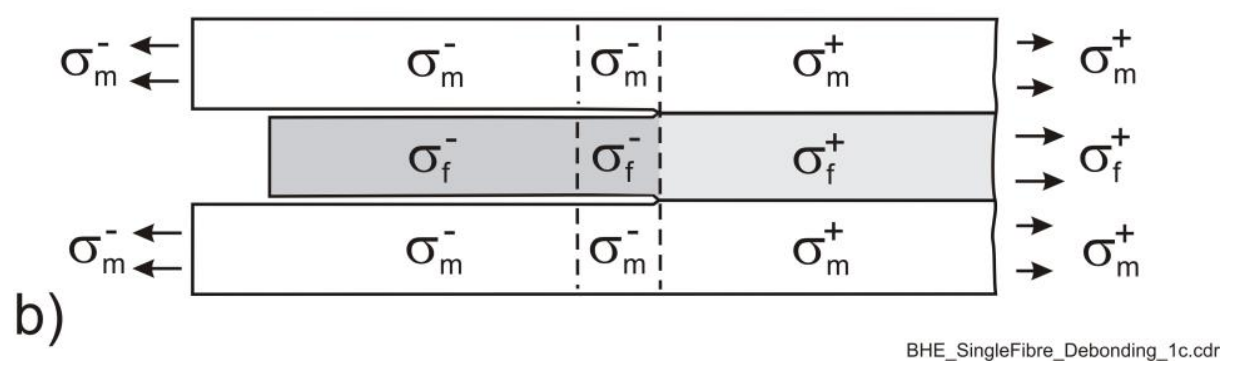

Fig. 3: The present problem recast in the framework of the BHE approach. State I (a) refers to the situation with a debond length, $\ell_{d}$, and State II (b) refers to a situation with a debond length $\ell_{d}+\Delta \ell_{d}$, where $\Delta \ell_{d}$ is a small extension of the debond crack. This is the volume in which there is a change in the stress state. 
Neglecting higher order terms of $\Delta \ell_{d}$ (since $\Delta \ell_{d}<<\ell_{d}$ ) leads to

$$
\begin{aligned}
\Pi_{I}-\Pi_{I I}= & \frac{\pi}{2} r^{2}\left\{4 \frac{E_{c}}{E_{f}} \frac{1}{\left(1-V_{f}\right) E_{m}} \tau_{s}^{2}\left(\frac{\ell_{d}}{r}\right)^{2}+4\left(\Delta \varepsilon^{T}-\frac{\bar{\sigma}}{\left(1-V_{f}\right) E_{m}}\right) \tau_{s}\left(\frac{\ell_{d}}{r}\right)\right. \\
& \left.+\frac{E_{f}}{E_{c}} \frac{1}{\left(1-V_{f}\right) E_{m}}\left(\left(1-V_{f}\right) E_{m} \Delta \varepsilon^{T}-\bar{\sigma}\right)^{2}\right\} \Delta \ell_{d}+\xi_{F} .
\end{aligned}
$$

The potential energy loss will be available for the energy absorption by the debonding of the interface crack tip and by the frictional energy dissipation within the frictional slipping zone. More precisely, debond crack propagation will occur only when the potential energy loss is equal to (or greater than) the energy dissipation. This can be written as:

$$
\Pi_{I}-\Pi_{I I}=\mathscr{g}_{c}^{i} 2 \pi r \Delta \ell_{d}+\xi_{F}
$$

where $\mathscr{G}_{c}^{i}$ is the critical energy release rate (fracture energy) of the debond crack tip of the fiber/matrix interface.

Next, the right hand side of equation (16) is inserted in the left hand side of equation (17). Then the term $\xi_{F}$ appears on both sides of the equation and thus cancels out. Furthermore, $\Delta \ell_{d}$ appears on all remaining terms and cancels out too. Dividing with $E_{f} r^{2} / 2$ on both sides puts the equation (17) into non-dimensional form:

$$
\begin{gathered}
\frac{4 E_{c}}{\left(1-V_{f}\right) E_{m}}\left(\frac{\tau_{s}}{E_{f}}\right)^{2}\left(\frac{\ell_{d}}{r}\right)^{2}+4\left(\Delta \varepsilon^{T}-\frac{\bar{\sigma}}{\left(1-V_{f}\right) E_{m}}\right)\left(\frac{\tau_{s}}{E_{f}}\right)\left(\frac{\ell_{d}}{r}\right) \\
+\frac{\left(1-V_{f}\right) E_{m}}{E_{c}}\left(\Delta \varepsilon^{T}-\frac{\bar{\sigma}}{\left(1-V_{f}\right) E_{m}}\right)^{2}-4 \frac{\mathscr{G}_{c}^{i}}{E_{f} r}=0 .
\end{gathered}
$$

To proceed further, we need to consider two cases, $\tau_{s}=0$ and $\tau_{s} \neq 0$. First, for $\tau_{s}=0, \ell_{d} / r$ vanishes from eq. (18). The implication is that when $\tau_{s}=0$, the debond 
length is unbounded; debonding will occur along the entire length of the fiber. For $\tau_{s}=0$, eq. (18) becomes

$$
\frac{\bar{\sigma}}{\left(1-V_{f}\right) E_{m}}-\Delta \varepsilon^{T}= \pm 2 \sqrt{\frac{E_{c}}{\left(1-V_{f}\right) E_{m}}\left(\frac{\mathscr{G}_{c}^{i}}{E_{f} r}\right)} .
$$

Since, on physical ground, with all other parameters held fixed, a higher value of $\mathscr{U}_{c}^{i}$ is expected to result in a higher stress level for debonding, the minus sign ahead of the square root should be dropped. The result is then identical to the results found by Pupurs and Varna (2013).

For $\tau_{s} \neq 0$, equation (18) is a second order equation in $\ell_{d} / r$. The solution is

$$
\frac{\ell_{d}}{r}=\frac{E_{f}}{2 \tau_{s}}\left(\frac{\bar{\sigma}}{E_{c}}-\frac{\left(1-V_{f}\right) E_{m}}{E_{c}} \Delta \varepsilon^{T}\right) \pm \frac{E_{f}}{\tau_{s}} \sqrt{\frac{\left(1-V_{f}\right) E_{m}}{E_{c}}\left(\frac{\mathscr{G}_{c}^{i}}{E_{f} r}\right)} .
$$

On physical grounds, we expect, with all other parameters fixed, the debond length to be lower for higher values of $\mathscr{G}_{c}^{i}$. Therefore, the plus sign in (20) must be dropped:

$$
\frac{\ell_{d}}{r}=\frac{E_{f}}{2 \tau_{s}}\left(\frac{\bar{\sigma}}{E_{c}}-\frac{\left(1-V_{f}\right) E_{m}}{E_{c}} \Delta \varepsilon^{T}\right)-\frac{E_{f}}{\tau_{s}} \sqrt{\frac{\left(1-V_{f}\right) E_{m}}{E_{c}}\left(\frac{\mathscr{G}_{c}^{i}}{E_{f} r}\right)} .
$$

\section{$\underline{4.3 \text { Model results: Stress-debond length relations }}$}

In the following, we re-write the derived equations to more operative forms. Equation (21) can be rewritten to express $\bar{\sigma}$ as a function of $\ell_{d}$. The result is:

$$
\frac{\bar{\sigma}}{E_{c}}=\frac{\left(1-V_{f}\right) E_{m}}{E_{c}} \Delta \varepsilon^{T}+2 \sqrt{\frac{\left(1-V_{f}\right) E_{m}}{E_{c}}\left(\frac{\mathscr{G}_{c}^{i}}{E_{f} r}\right)}+2 \frac{\tau_{s}}{E_{f}}\left(\frac{\ell_{d}}{r}\right) .
$$

Following Hutchinson and Jensen (1990), we introduce the debond initiation stress, $\bar{\sigma}_{i}$, defined as 


$$
\frac{\bar{\sigma}_{i}}{E_{c}}=\frac{\left(1-V_{f}\right) E_{m}}{E_{c}} \Delta \varepsilon^{T}+2 \sqrt{\frac{\left(1-V_{f}\right) E_{m}}{E_{c}}\left(\frac{\mathscr{\vartheta}_{c}^{i}}{E_{f} r}\right)} .
$$

In case of a zero friction the debond crack will propagate along the entire fiber/matrix interface at this stress value; note that (23) is identical to (19). Having introduced $\bar{\sigma}_{i}$, equation (22) can be written as

$$
\frac{\bar{\sigma}}{E_{c}}=\frac{\bar{\sigma}_{i}}{E_{c}}+2 \frac{\tau_{s}}{E_{f}}\left(\frac{\ell_{d}}{r}\right)
$$

It is clear from (22) and (24) that $\bar{\sigma}$ is related linearly to $\ell_{d}$.

\section{Determination of fiber opening displacements}

\section{$\underline{5.1 \text { Analysis }}$}

Next, we determine the opening displacement of the broken fiber. In the present analysis, the opening displacement, $\delta$, is obtained by integration of the strain differences of the matrix and the fiber in the debonded zone, $0 \leq z \leq \ell_{d}$,

$$
\delta=\int_{0}^{\ell_{d}}\left(\varepsilon_{m}^{-}(z)-\varepsilon_{f}^{-}(z)\right) d z
$$

where the strains are obtained from Hooke's law:

$$
\varepsilon_{m}^{-}(z)=\frac{\sigma_{m}^{-}(z)}{E_{m}}+\varepsilon_{m}^{T} \quad \text { and } \quad \varepsilon_{f}^{-}(z)=\frac{\sigma_{f}^{-}(z)}{E_{f}}+\varepsilon_{f}^{T}
$$

and $\varepsilon_{m}^{T}$ and $\varepsilon_{f}^{T}$ are the in-elastic strain of the fiber and matrix respectively, while $\sigma_{m}^{-}(z)$ and $\sigma_{f}^{-}(z)$ are given by (9) and (10), respectively. Inserting (26) into (25), using (3), (9) and (10) and performing the integration leads to

$$
\frac{\delta}{r}=\left(\frac{\bar{\sigma}}{\left(1-V_{f}\right) E_{m}}-\Delta \varepsilon^{T}\right)\left(\frac{\ell_{d}}{r}\right)-\frac{E_{c}}{\left(1-V_{f}\right) E_{m}} \frac{\tau_{s}}{E_{f}}\left(\frac{\ell_{d}}{r}\right)^{2} .
$$


It can be seen from (27) that the fiber opening displacement increases non-linearly with increasing debond length and with increasing applied stress. An increase in $\ell_{d}$ increases the part where slip occurs. An increase in $\bar{\sigma}$ increases the strain in the matrix in the debonded zone and thus increases the relative displacement difference, since the stress (and thus the strain) in the debonded fiber $\sigma_{f}^{-}(z)$ remains the same, being controlled by $\tau_{s}$.

Inserting $\ell_{d}$ from (21) into (27) leads to

$$
\frac{\delta}{r}=\frac{1}{4} \frac{E_{f}}{\tau_{s}} \frac{\left(1-V_{f}\right) E_{m}}{E_{c}}\left(\frac{\bar{\sigma}}{\left(1-V_{f}\right) E_{m}}-\Delta \varepsilon^{T}\right)^{2}-\frac{\mathscr{G}_{c}^{i}}{\tau_{s} r} .
$$

Rewriting (28), we can express $\bar{\sigma}$ as a function of $\delta$. The result is

$$
\frac{\bar{\sigma}}{E_{c}}=\frac{\left(1-V_{f}\right) E_{m}}{E_{c}} \Delta \varepsilon^{T}+2 \sqrt{\frac{\left(1-V_{f}\right) E_{m}}{E_{c}} \frac{\tau_{s}}{E_{f}}\left(\frac{\delta}{r}+\frac{\mathscr{\vartheta}_{c}^{i}}{\tau_{s} r}\right)} .
$$

It is also useful to isolate $\Delta \varepsilon^{T}$ from (27). The result is:

$$
\Delta \varepsilon^{T}=\left\{\frac{\bar{\sigma}}{E_{c}}-\frac{\tau_{s}}{E_{f}} \frac{\ell_{d}}{r}\right\} \frac{E_{c}}{\left(1-V_{f}\right) E_{m}}-\frac{\delta}{\ell_{d}} .
$$

This result is independent of $\mathscr{S}_{c}^{i}$.

Finally, by setting $\ell_{d}=0$ in (28) (so that per definition $\bar{\sigma}=\bar{\sigma}_{i}$ ) we can isolate $\mathscr{G}_{c}{ }^{i}$ :

$$
\frac{\mathscr{G}_{c}^{i}}{E_{f} r}=\frac{1}{4} \frac{\left(1-V_{f}\right) E_{m}}{E_{c}}\left(\frac{\bar{\sigma}_{i}}{\left(1-V_{f}\right) E_{m}}-\Delta \varepsilon^{T}\right)^{2}
$$

This result is independent of $\tau_{s}$. Inserting $\Delta \varepsilon^{T}$ from (30) into (31) gives

$$
\frac{\mathscr{G}_{c}^{i}}{E_{f} r}=\frac{1}{4} \frac{\left(1-V_{f}\right) E_{m}}{E_{c}}\left(\frac{\bar{\sigma}-\bar{\sigma}_{i}}{\left(1-V_{f}\right) E_{m}}-\frac{E_{c}}{\left(1-V_{f}\right) E_{m}} \frac{\tau_{s}}{E_{f}}\left(\frac{\ell_{d}}{r}\right)-\frac{\delta}{\ell_{d}}\right)^{2} .
$$


This result is independent of $\Delta \varepsilon^{T}$. Eq. (31) and (32) provide two alternative ways to determine $\mathscr{G}_{c}^{i}$. Which of the equations is preferred in the calculation of $\mathscr{G}_{c}^{i}$ depends on which of the parameters $\tau_{s}$ and $\Delta \varepsilon^{T}$ are determined with the highest accuracy.

\section{$\underline{5.2 \text { Approximate solutions for }} V_{f} \rightarrow 0$}

In many cases, specimens for single-fiber fragmentations tests are made with dimensions such that $V_{f} \approx 0$. It is therefore convenient to develop approximate solutions for $V_{f} \rightarrow 0$. First, from (7) we note that

$$
E_{c} \rightarrow E_{m} \quad \text { for } \quad V_{f} \rightarrow 0
$$

Then (22) reduces to

$$
\frac{\bar{\sigma}}{E_{m}}=\Delta \varepsilon^{T}+2 \sqrt{\frac{\mathscr{G}_{c}^{i}}{E_{f} r}}+2 \frac{\tau_{s}}{E_{f}} \frac{\ell_{d}}{r} .
$$

$\bar{\sigma}$ can be predicted as a function of $\delta$ by (29) which for $V_{f} \rightarrow 0$ approaches

$$
\frac{\bar{\sigma}}{E_{m}}=\Delta \varepsilon^{T}+2 \sqrt{\frac{\tau_{s}}{E_{f}}\left(\frac{\delta}{r}+\frac{\mathscr{G}_{c}^{i}}{\tau_{s} r}\right)}
$$

while (23) reduces to

$$
\frac{\bar{\sigma}_{i}}{E_{m}}=\Delta \varepsilon^{T}+2 \sqrt{\frac{\mathscr{G}_{c}^{i}}{E_{f} r}} .
$$

Likewise for $V_{f} \rightarrow 0,(30)$ becomes

$$
\Delta \varepsilon^{T}=\frac{\bar{\sigma}}{E_{m}}-\frac{\tau_{s}}{E_{f}} \frac{\ell_{d}}{r}-\frac{\delta}{\ell_{d}}
$$

and (31) becomes

$$
\frac{\mathscr{G}_{c}^{i}}{E_{f} r}=\frac{1}{4}\left(\frac{\bar{\sigma}_{i}}{E_{m}}-\Delta \varepsilon^{T}\right)^{2},
$$


while eq. (32) reduces to

$$
\frac{\mathscr{G}_{c}^{i}}{E_{f} r}=\frac{1}{4}\left(\frac{\bar{\sigma}-\bar{\sigma}_{i}}{E_{m}}-\frac{\tau_{s}}{E_{f}}\left(\frac{\ell_{d}}{r}\right)-\frac{\delta}{\ell_{d}}\right)^{2} .
$$

These equations are relative brief and are thus fairly easy to use.

\section{Model application}

\section{$\underline{6.1 \text { Model verification }}$}

In this section we test our 1-D model with the more accurate results from the numerical BEM model of Graciani et al. (2009). Using the material data of their paper, we first estimate $\bar{\sigma}_{i}$ by eq. (23) using a value of $\mathscr{\varphi}_{c}^{i}=50 \mathrm{~J} / \mathrm{m}^{2}$ given in the paper. In case $\Delta \varepsilon^{T}=0$ we get $\bar{\sigma}_{i} / E_{m}=2.39 \%$. From Fig. 6 in Graciani et al., (2009) we read off $\bar{\sigma}_{i} / E_{m}=2.39 \%$. Including residual stresses (calculating $\Delta \varepsilon^{T}$ from the data listed in Graciani et al. (2009)), (23) gives $\bar{\sigma}_{i} / E_{m}=2.73 \%$. From the figure in Graciani et al., (2009) we read off $\bar{\sigma}_{i} / E_{m}=2.76 \%$. From the slope of the linear part of the curve $\left(\ell_{d} / r>5\right)$ we can calculate $\tau_{s}$ from (34)

$$
\frac{d\left(\bar{\sigma} / E_{m}\right)}{d\left(\ell_{d} / r\right)}=2 \frac{\tau_{s}}{E_{m}} \quad \Rightarrow \tau_{s}=\frac{E_{f}}{2} \frac{d\left(\bar{\sigma} / E_{m}\right)}{d\left(\ell_{d} / r\right)}
$$

The result is $\tau_{s}=20.9 \mathrm{MPa}$. Unfortunately, only the Coulomb friction coefficient $(\mu=$ 0.3) is given in the paper of Graciani et al. (2009) so it is not possible to compare the shear stress values. Next, an extrapolation of the linear part of the curve $\left(\ell_{d} / r>5\right)$ to $\ell_{d} / r=0$ gives a value of $\bar{\sigma}_{i} / E_{m}$ as $0.379 \%$, and with $r=8 \mu \mathrm{m}$, we then calculate

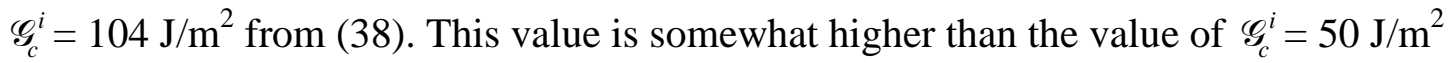


used in the simulations. This suggests that the simple model developed in this paper tends to overestimate $\mathscr{G}_{c}^{i}$.

\section{$\underline{6.2 \text { Model prediction }}$}

A few examples are now given of model predictions. We take $V_{f}=0$, so that the equations in Section 5.2 apply. In the predictions, we use $E_{m}=3 \mathrm{GPa}, E_{f}=70 \mathrm{GPa}$ and $r=8 \mu \mathrm{m}$, values appropriate for a glass fiber composites (Graciani et al., 2009).

Model predictions, made using (35), are shown in Fig. 4. The relationship between the applied stress, $\bar{\sigma}$, and the opening displacement, $\delta$, are shown for various values of the interfacial fracture energy, $\mathscr{G}_{c}^{i}$, and the frictional sliding shear stress, $\tau_{s}$ with $\Delta \varepsilon^{T}=0$. The values shown are expected to cover the realistic range for glass fiber composites. It is seen that for a fixed value of $\tau_{s}$, a higher value of $\mathscr{G}_{c}^{i}$ gives a higher value of $\bar{\sigma}$ at the same opening, $\delta$, while the slope of the $\bar{\sigma}-\delta$ curve is lower. Furthermore, a higher value of $\tau_{s}$ gives a higher value of $\bar{\sigma}$ for the same value of $\delta$ and a higher the slope of the $\bar{\sigma}-\delta$. We note - as also found experimentally by Kim and Nairn (2002a) - that opening displacements of several microns are realistic to measure by optical microscopy.

Fig. 5 shows model predictions for $\bar{\sigma}_{i}$, calculated for various values of $\mathscr{G}_{c}^{i}$ and $\tau_{s}$ using (36). $\bar{\sigma}_{i}$ increases with increasing $\mathscr{G}_{c}^{i}$. Also, a higher value of $\Delta \varepsilon^{T}$ results in a higher value of $\bar{\sigma}_{i}$. The curves have the same shape, but they are translated. 


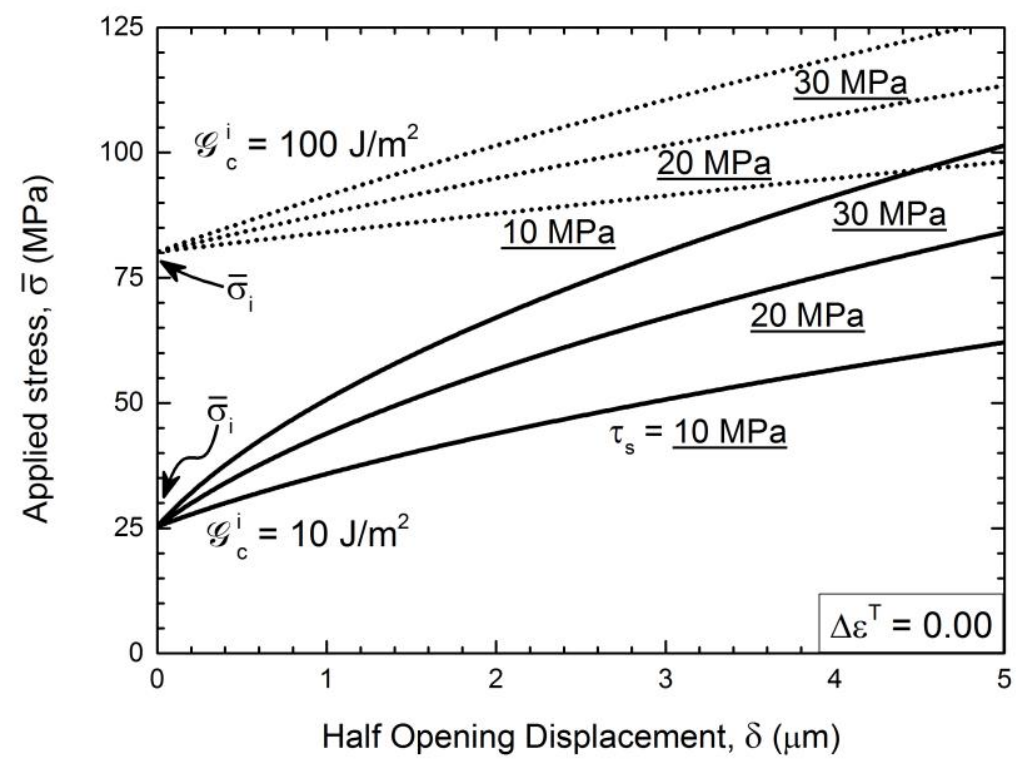

Fig. 4: Predicted behavior of single broken glass fiber embedded in an epoxy matrix. Stress-fiber opening relationship for various interfacial parameters.

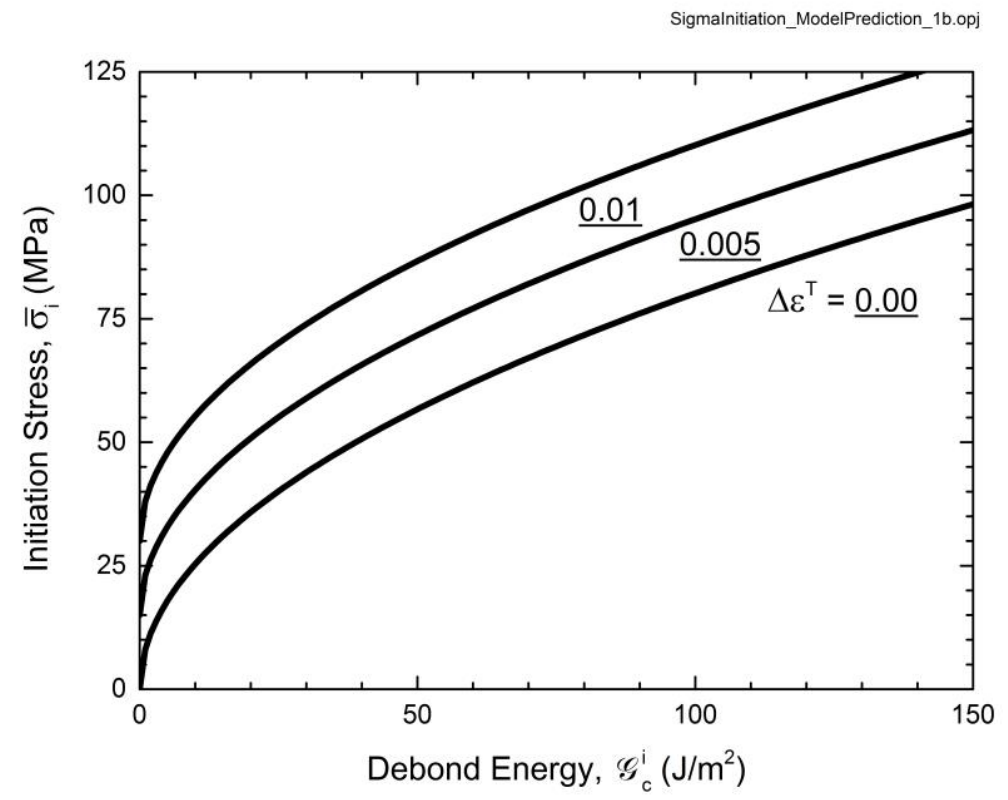

Fig. 5: Model prediction of stress at debond initiation as a function of interfacial fracture energy and mismatch strain for a glass fiber embedded in an epoxy matrix. 
For both Fig. 4 and Fig. 5 we find that the curves for different parameters are fairly distinct, suggesting that it should be possible to determine $\mathscr{G}_{c}^{i}$ and $\tau_{s}$ fairly accurately from experimental data.

\subsection{An approach for determination of interface parameters}

In the following we describe an approach for the determination of the interface parameters $\mathscr{G}_{c}^{i}$ and $\tau_{s}$ from measurement from a single fiber fragmentation test. It is assumed that the following parameters are known before the experiment is conducted:

$$
E_{f}, E_{m}, V_{f}, r
$$

The following parameters are measured simultaneously during the single fiber fragmentation test (in this process, only data for $\ell_{d} / r>5$ should be use):

$$
\bar{\sigma}, \delta, \ell_{d}
$$

More precisely, we wish to record simultaneous values of $\ell_{d}$ and $\delta$ as a function of $\bar{\sigma}$. For transparent matrix materials such as thermosetting polymer matrix materials, consecutive values of $\ell_{d}$ and $\delta$ can be determined e.g. from micrographs recorded using optical miscopy during the experiments.

The three microscale parameters that we need to determine from the analysis are:

$$
\mathscr{G}_{c}^{i}, \tau_{s}, \Delta \varepsilon^{T}
$$

We propose the following approach to determine the three parameters listed in (42) one by one, i.e., in three steps: 
Step 1: $\bar{\sigma}$ is plotted as a function of $\ell_{d}$. Since, according to (22) and (34), $\bar{\sigma}$ should depend linearly on $\ell_{d}$, a straight line can be fitted to the data. This allows the determination of $\tau_{s}$. A sketch of such a plot is shown in Fig. 6a.

Step 2: Having determined $\tau_{s}$, a plot of data for the experimental values of $\bar{\sigma}, \delta$ and $\ell_{d}$ is constructed according to (30) or (37). The result should be a constant value, $\Delta \varepsilon^{T}$. An average value of $\Delta \varepsilon^{T}$ can be determined from such data. A sketch of such a plot is shown in Fig. 6 b.

Step 3: Knowing $\tau_{s}$, the value of $\mathscr{G}_{c}^{i}$ can be determined by the use of (31) - (32) or (38) - (39), e.g. from a graph showing $\mathscr{G}_{c}^{i}$ as a function of $\bar{\sigma}$ using the associated values of $\ell_{d}$ and $\delta$. A sketch of such a plot is shown in Fig. 6c.

This approach enables us to determine the three parameters $\tau_{s}, \Delta \varepsilon^{T}$ and $\mathscr{G}_{c}^{i}$ sequentially. No knowledge of $\Delta \varepsilon^{T}$ and $\mathscr{G}_{c}^{i}$ is required to determine $\tau_{s}$ (Step 1). Moreover, only $\tau_{s}$ needs to be known to determine $\Delta \varepsilon^{T}$ (Step 2) and $\mathscr{G}_{c}^{i}$ (Step 3). A consistency check on $\mathscr{G}_{c}^{i}$ can be made as follows. First, the value of $\bar{\sigma}_{i}$ is obtained from a graph showing $\bar{\sigma}$ as a function of $\ell_{d}$ (as Fig. 6a) by extrapolation to $\ell_{d}=0$ (it is emphasized that the model, being a shear-lag model, should not be used for short debonds, i.e., for data where $\ell_{d} / r<5$ and certainly not for data associated with debond initiation). Having determined $\bar{\sigma}_{i}$ and $\Delta \varepsilon^{T}$, an independent value of $\mathscr{G}_{c}^{i}$ can 
be determined from (31) or (38). Furthermore, having identified values of $\mathscr{\varphi}_{c}^{i}, \tau_{s}$ and $\Delta \varepsilon^{T}$, we can make a another check by plotting $\bar{\sigma}$ as a function of $\delta$, using eq. (22) or (34), and compare the outcome with the original experimental data for $\bar{\sigma}$ as a function of $\delta$.

\subsection{Application of model on experimental data}

It appears to be no published experimental data where data for applied stress, debond length and broken fibre gap are presented. Thus, the complete model approach cannot be tested. However, the data from Kim and Nairn (2002b) can be analyzed by the present model. Their data were for new debonds only. Some of the data points for the highest applied strain values might be from fiber breaks that are so closely spaced that they interact but are still used in the following, despite that the present model assumes that the fiber breaks behave as isolated breaks (i.e., with no interaction). However, since the relationship between debond length and is only expected to be linear for $\ell_{d} / r>5$ (Hutchinson and Jensen, 1990; Graciani et al., 2009), only data for $\ell_{d} / r>$ 5 are used in the following.

First, lines were fitted to the data as "best fit", as well as lines having the lowest and highest slopes going through the experimental data for the applied strain and debond length (reproduced in Fig. 7). The value of $\tau_{s}$ is estimated from the slope of the fitted lines, using (40) with $E_{f}=72.5 \mathrm{GPa}$. The frictional shear stress was calculated to be $\tau_{s}=33 \mathrm{MPa}$ (best fit), with $13 \mathrm{MPa}$ and $48 \mathrm{MPa}$ as lowest and highest values. For comparison, we can estimate the Coulomb friction shear stress as follows (Kim and Nairn, 2002b) using $\psi_{f}=0.01$ : 

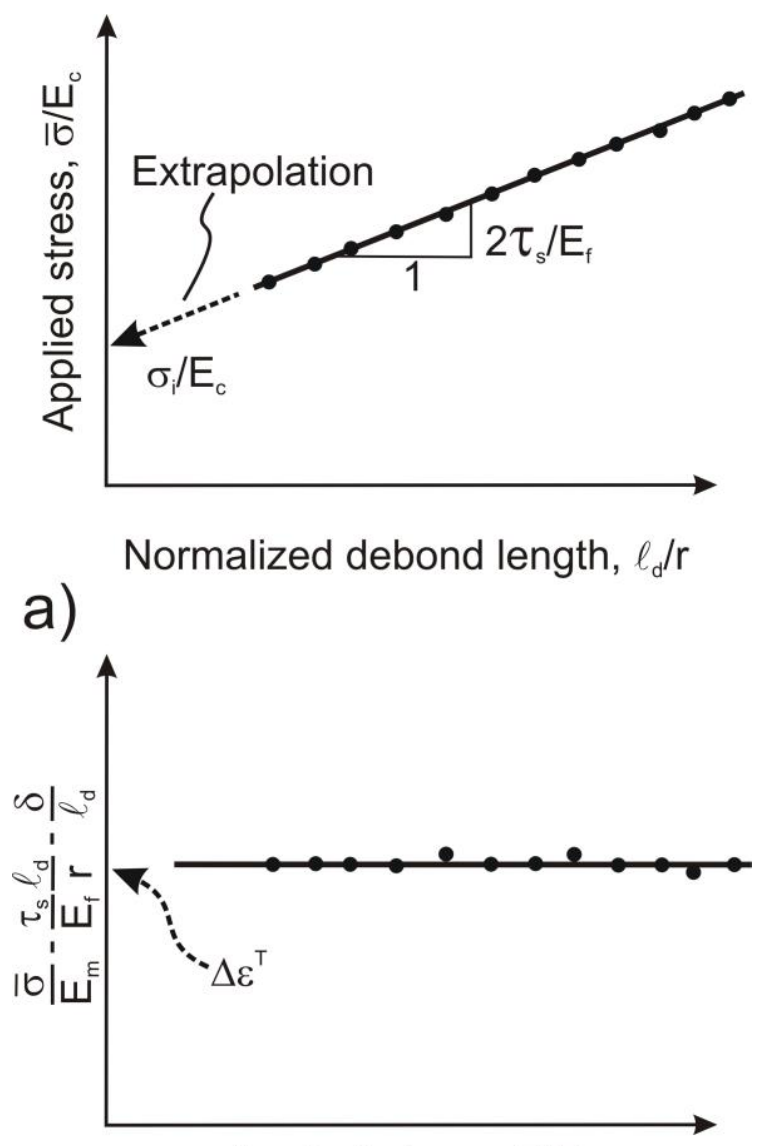

Applied stress, $\bar{\sigma} / \mathrm{E}_{\mathrm{m}}$

b)

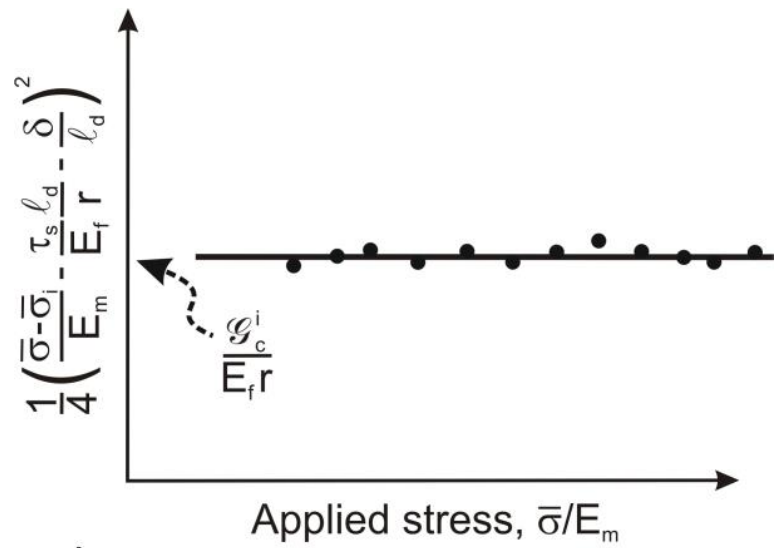

c)

Fig. 6: Schematics of approach for determination of microscale parameters from experiments. A plot of $\bar{\sigma}$ as a function of $\ell_{d}$ for the determination of $\tau_{s}$, (a). Plot of experimental values of $\bar{\sigma}, \delta$ and $\ell_{d}$ combined according to (37) and (39) to give $\Delta \varepsilon^{T}(\mathrm{~b})$ and $\mathscr{G}_{c}^{i}$ as a function of $\bar{\sigma}(\mathrm{c})$. 


$$
\tau_{f}=\psi_{f}\left(\bar{\sigma} / E_{m}-\Delta \varepsilon^{T}\right) E_{f} .
$$

This gives $\tau_{f} \approx 12 \mathrm{MPa}$ for $\bar{\sigma} / E_{m}=2 \%$ and $20 \mathrm{MPa}$ for $\bar{\sigma} / E_{m}=3.0 \%$. These values are lower than the "best fit" value of $\tau_{s}$ found by the new proposed approach, but almost within the range of the lowest and highest values.

Next, by extrapolation the lines for data for $\ell_{d} / r>5$ to $\ell_{d} / r=0$, we identify lower, best and highest values for $\bar{\sigma}_{i} / E_{m}$. With no data available for the fiber break gap, no value of $\Delta \varepsilon^{T}$ can be calculated from the experimental data. Therefore, $\Delta \varepsilon^{T}$ was estimated from the data in the paper and eq. (5) to 0.00346 . Then, $\mathscr{G}_{c}^{i}$ was calculated from (38) giving $27 \mathrm{~J} / \mathrm{m}^{2}$ for best fit and $14 \mathrm{~J} / \mathrm{m}^{2}$ and $50 \mathrm{~J} / \mathrm{m}^{2}$ as lowest and highest values. These values of $\mathscr{G}_{c}^{i}$ are significantly lower than the value of $120 \mathrm{~J} / \mathrm{m}^{2}$ found by Kim and Nairn (2002b), but only slightly higher than the value of $12 \mathrm{~J} / \mathrm{m}^{2}$ found by Graciani et al. (2011).

Since the values of $\tau_{s}$ are directly related to the slope of the lines in Fig. 7, it is possible to assess the realism of $\tau_{s}$ values visually. For instance, it can immediately be seen from Fig. 7 that the dotted lines that represent upper and lower bounds of the data have slopes and thus values of $\tau_{s}$ and $\bar{\sigma}_{i} / E_{m}$ that are in between the lowest and highest values calculated from the thin solid lines.

\section{Discussion}

\section{$\underline{7.1 \text { Model approximations }}$}

The present analysis is based on three major assumptions: First, materials are taken to be linear elastic. Second, the analysis is one-dimensional and neglects Poisson's 
effects, and third, the frictional response of the fiber/matrix is described in terms of a constant shear stress, $\tau_{s}$. These assumptions enable the development of a closed form analytical solution and thus leads to an approach and equations that are relative easy to use. However, it is appropriate to discuss this assumptions in some details.

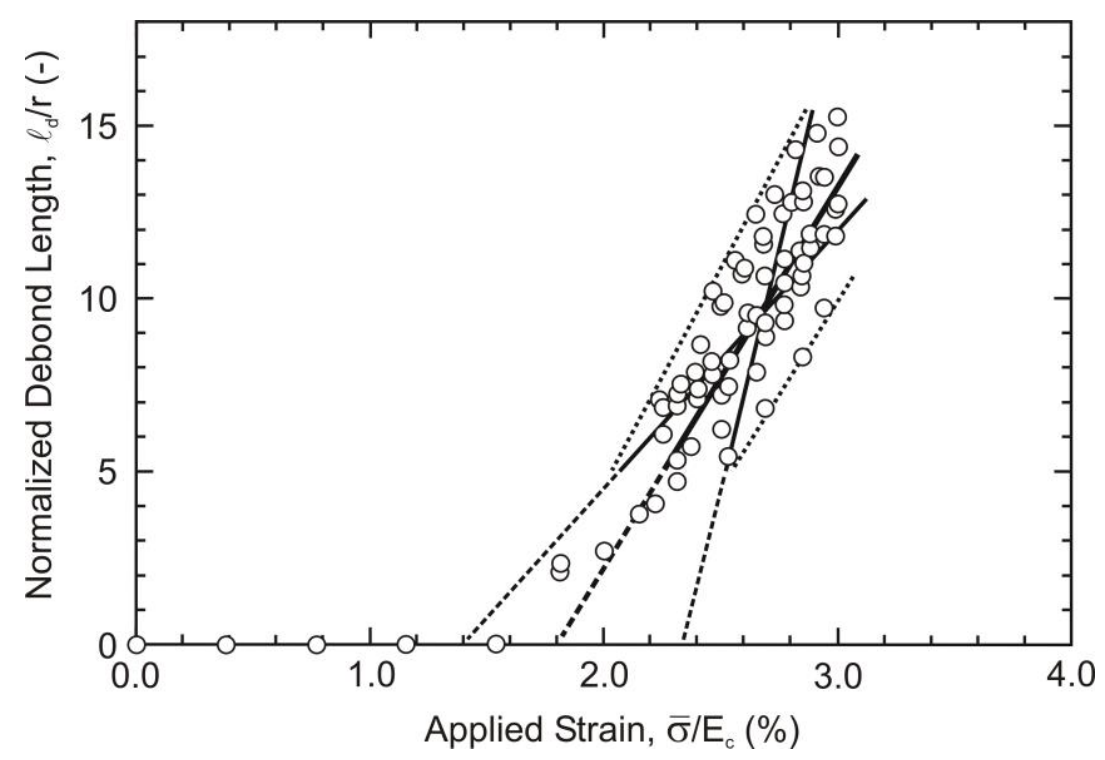

Fig. 7: Normalized debond length as a function of applied strain for a glass fiber in an epoxy matrix - data from Kim and Nairn (2002b). Thick line indicates best fit to data for $\ell_{d} / r>5$; the thin lines have the lowest and highest slopes. Dashed lines are extrapolations to $\ell_{d} / r=0$. Dotted lines indicate upper and lower bounds to the data.

While linear-elastic behavior is a good assumption for most fibers, many polymer materials that are used as matrix material in fiber composites have a non-linear stressstrain behavior. It is likely therefore, that such material develop plasticity, in particularly at the debond crack tip. If the plastic zone size is small (small-scale yielding) the toughening enhancement due to crack tip plasticity may be taken as a part of the engineering interfacial fracture energy. On the other hand, it has also been shown for thin plastic layers that when the layer thickness of the plastic material is 
decreased - corresponding to closely spaced fibers in a composite material - the effect of plasticity is significantly reduced (Tvergaard and Hutchinson, 1994). Thus, the effect of plasticity on fiber/matrix debonding in a single filament composite specimen may be different from that in a composite material with closely spaced fibers (higher fiber volume fraction). This issue deserves to be studied further.

The present shear-lag model is one-dimensional in the sense that it includes only the normal stress in the $z$-direction which is taken to be uniform across the cross section of the fiber and across the matrix; radial and hoop stresses are ignored and stress equilibrium is only satisfied in the average sense. Such approximations are frequently made in analytical micromechanical models (Marshall et al., 1985; Budiansky et al., 1986; Budiansky et al., 1995). Using a more sophisticated shear-lag model, Hutchinson and Jensen (1990) showed that in comparisons with an accurate numerical solution, shear-lag models can be good approximations for energy release rate and fiber end displacements once $\ell_{d}$ is longer than about five times $r$. The existing 3Dmodels of the single fiber fragmentation problem (Wagner et al., 1995; Wu et al., 2000; Graciani et al., 2009) are obviously more accurate. But the solutions are lengthy and unrevealing and are not easy to use. Thus, the present 1-D shear lag model seems to be a fair balance between accuracy and accessible.

\subsection{Remarks on description of friction}

The mechanics description of interfacial sliding is an un-resolved issue. With Coulomb friction, the frictional shear stress is taken to be proportional to a friction coefficient and the normal pressure acting normal to the interface. The normal pressure will in general depend on the Poisson's contraction of the fiber and matrix as 
well as interface roughness asperities (Pathasarathy et al., 1991). However, neither the Poisson's ratio nor the interface roughness asperities are known for most engineering fibers. It has been proposed that the effect of Poisson's contraction can outbalance the effect of roughness asperities (Marshall et al., 1992). The mixed friction law (1) seems to give a reasonably representation since the second term incorporates the radial normal stress, $\sigma_{r r}$ which will depend on temperature in case the fiber and matrix have different thermal expansion coefficients. This enables the prediction of as a function of temperature (Connell and Zok, 1997). Cohesive laws can also be formulated to mimic roughness asperities (Sørensen and Goutianos, 2014). Good experiments are needed to guide to the development of the best choice of frictional law. At the present state a constant shear stress seems to be a fair assumption for the analysis of experiments.

\subsection{Transferability of parameters to real composites}

It is relevant to consider how parameters measured at single filament composites can be transferred to models of real fiber composites with fiber volume fraction of about 30-60\%. First, the mismatch strain $\Delta \varepsilon^{T}$, determined from a single filament specimen with $V_{f} \approx 0$, can directly be used in a micromechanical model, e.g. (6), to calculate the residual stresses in a composite with $V_{f} \approx 40-60 \%$. Second, the interfacial fracture energy is taken to be the Mode II (meaning that the crack faces displaces tangentially) fracture energy of the fiber/matrix, and thus be a property of the bi-material interface, independent of $V_{f}$. Third, $\tau_{s}$ probably cannot be taken to be an interface property independent of $V_{f}$. As indicated by (1), the interfacial sliding frictional shear stress in a composite is likely to depend on both fiber roughness, and Coulomb friction, i.e. 
depends on both a friction coefficient $\mu$ and the radial normal stress $\sigma_{r r}$ acting normal to the debonded fiber/matrix interface.

At the present there appears to be no consensus about the most appropriate way of modelling friction sliding stress. Therefore, further research is needed to investigate how the frictional shear stress in a real composite depends on e.g. fiber volume fraction and radial normal stress across the interface. The most important outcome of the present model is that it enables a clear separation between interfacial fracture energy (breakage of chemical bonds) and interfacial sliding shear stress in a robust way, which the previous approach (not measuring the fiber gap) did not enable. Experiments of the type proposed here could be conducted for specimens at various temperatures in order to assess how the measured values of $\tau_{s}$ would depend on interfacial normal stresses. Other types of experiments on composites, such as the single fiber pull-out and fiber push-out experiments conducted by Marshall et al. (1992) could be used to assess the frictional sliding shear stress in composites with high fiber volume fraction.

In the area of the development of improved composite materials, an important issue is to understand and control the interfacial chemistry (fiber coating/sizing) which will affect debonding and possibly to a less extend the frictional sliding responds (roughness, friction coefficient). A robust tool - like the present model - that enables a clear separation of the two mechanical parameters will be very valuable, because experiments of the type proposed here are expected to show the correct trends even though the measured value of $\tau_{s}$ obtained from single filament composite specimens might not to be identical to the frictional shear stress in a composite with high fiber 
volume fraction. Surface treatments that lead to increasing interface roughness or and increasing friction coefficient are likely to increase the frictional shear stress for both the single filament and the "real" composite. Thus, single filament composite specimens will still be a valuable tool in the development of new fiber surface treatments.

Another point is that for multi-directional laminates, fibers inside a layer will often be subjected to tensile or compressive stress in-plane, perpendicular to the fiber direction, i.e. normal to the fiber/matrix interface. This again would likely affect frictional sliding behavior; additional (numerical) modelling would then be required to study effects on frictional sliding and interfacial fracture energy. But if the interfacial fracture energy and the frictional sliding interface law (including Coulombic effects) were obtained from various experiments conducted at single filament composite specimens, the effect of multiaxial stress state could be accounted for by micromechanical modelling. The area of characterization and modelling of the frictional behavior of the fiber /matrix interface deserves more investigations.

\subsection{Traditional interpretation of single fiber fragmentation tests}

The traditional way of analyzing data from single fiber fragmentation tests is to calculate the interfacial shear stress from the length of fragmented fibers (i.e., the $z-$ distance between two fiber breaks) in the saturated state, where the fibers are fully debonded so that the stress transfer is controlled entirely by the frictional shear stress. This approach is well suited for investigations of the effects of e.g. surface roughness and sizing on the sliding behavior, but not effects of chemistry on the interfacial fracture energy and separate effects of different $\Delta \varepsilon^{T}$ in different matrix materials. In 
contrast, the approach presented in this paper enables the determination of $\mathscr{G}_{c}^{i}, \tau_{s}$ and $\Delta \varepsilon^{T}$, giving a more realistic description of the mechanics of the fiber/matrix interface.

Although the approach proposed in the present paper can be combined with the traditional approach, the proposed approach should only be used as long as the fiber breaks are so far apart that they can be considered being independent, i.e. as long as the slip zones and crack tip stress fields from the broken fiber ends are not overlapping.

\subsection{Experimental data for models}

The data presented by Kim and Nairn (2002ab) do not make a distinction between isolated fiber debonds and interacting debonds. These results therefore includes both data where the relationship between $\ell_{d}$ and $\bar{\sigma}$ is non-linear and linear, are therefore more blurred and are more difficult to interpret. In order to understand the experiments better, it would be useful in the future to report data for isolated fiber breaks and debonding separately from data from interacting fiber breaks. For isolated fiber breaks, $\ell_{d}$ is, according to all models, expected to increase linearly with $\bar{\sigma}$ for $\ell_{d} / r>5$.

\subsection{In-situ determination of fiber strength}

It has been suggested (Tripathi and Jones, 1998) that that in-situ fiber strength could be lower than the fiber strength of virgin fibers due to additional defects being introduced to the fibers during processing of the composite. Therefore, it would be of interest to compare in-situ fiber strength values found by the proposed approach with 
tensile strength values obtained from tensile testing of single fibers before composite manufacture. The present model enables the determination of the fibers tensile strength in-situ.

Having determined the mismatch strain, $\Delta \varepsilon^{T}$, it is easy to back-calculate the stress in the fiber at the instant it breaks by the use of (8). Denote the applied composite stress at the occurrence of fiber break by $\bar{\sigma}_{f b}$, the in-situ strength of the fiber, $\sigma_{f u}$, can estimated from

$$
\frac{\sigma_{f u}}{E_{f}}=-\Delta \varepsilon^{T} \frac{\left(1-V_{f}\right) E_{m}}{E_{c}}+\frac{\bar{\sigma}_{f b}}{E_{c}} .
$$

The strength data cannot be compared directly, since the single fiber gives only one strength value for its gauge length (i.e. the weakest point within the gauge section) while the single filament composite specimen will give more strength values for a similar specimen gauge length, and the stress in the fiber is not uniform in the single filament composite specimen (due to the stress transfer along the debond zones), see Curtin (1991) for a detailed discussion.

\section{Conclusions}

An analytical shear-lag model, developed using the formalism of the potential energy approach of Budiansky, Hutchinson and Evans, enables the determination of the mechanical properties of the fiber/matrix interface of unidirectional composites in terms of a interfacial fracture energy and a frictional sliding shear stress from experimental data of the applied stress, the debond length and opening displacement of broken fibers. The model also allows the determination of the residual stresses and in-situ fiber strength values from single fiber fragmentation tests. 


\section{Acknowledgements}

This study was partially funded by the "Danish Centre for Composite Structure and materials for Wind Turbines (DCCSM)", fund no. 09-067212 from the Danish Council for Strategic Research. Thanks to Dr. Hans Lilholt for useful discussions and thanks to both reviewers for providing critical reviews with many good suggestions.

\section{References}

Budiansky, B., Evans, A. G., Hutchinson, J. W., 1995. Fiber-matix debonding effects on cracking in aligned fiber ceramic composites. International Journal of Solids and Structures, 32, 315-28.

Budiansky, B., Hutchinson, J. W., Evans, A. G., 1986. Matrix fracture in fiberreinforced ceramics. J. Mech. Phys. Solids., 34, 167-78.

Connell, S. J., and Zok, F. W., 1997. Measurement of the cyclic bridging law in a titanium matrix composite and its application to simulating crack growth. Acta Mater., $45,5203-11$.

Curtin, W. A., 1991. Theory of mechanical properties of ceramic matrix composites. $J$. Am. Ceram. Soc., 74, 2837-45.

Eshelby, J. D., 1957. The determination of the elastic field of an ellipsoidal inclusion, and related problems. Proceedings of the Royal Society of London, 241, 376-396.

Feih, S., Wei, J. Kingshott, P. and Sørensen, B. F., 2005. The influence of fibre sizing on the strength and fracture toughness of glass fibre composites. Composites part A, 36, 245-55. 
Graciani, E., Mantic, V., Paris, F., Varna, J., 2009. Numerical analysis of debond propagation in the single fiber fragmentation test. Composites Science and Technology, 69, 2514-20.

Graciani, E., Varna, J., Mantic, V., Blazquez, A. and Paris, F., 2011. Evaluation of interfacial fracture toughness and friction coefficient in the single fiber fragmentation test. Procedia Engineering. 10, 2478-83.

Hutchinson, J. W., Jensen, H. M., 1990. Models of fiber debonding and pullout in brittle composites with friction. Mechanics of Materials. 9, 139-63.

Kessler, H., Schüller, T., Beckert, W., Lauke, B., 1999. A fracture-mechanics model of the microbond test with interface friction. Composites Science and Technology. 59, $2231-42$.

Kim, B. W., Nairn, J. A., 2002a. Observations of fiber fracture and interfacial debonding phenomena using the fragmentation tests in single fiber composites. Journal of Composite Materials. 36, 1825-58.

Kim, B. W., and Nairn, J. A., 2002b. Experimental verification of the effect of friction and residual stress on the analysis of interfacial debonding and toughness in single fiber composites. Journal of Materials Science, 37, 3965-72.

Kelly, A., Tyson, W. R., 1965. Tensile properties of fiber-reinforced metals: Copper/Tungsten and Copper/Molybdenum. Journal of the Mechanics and Physics of Solids, 13, 329-50.

Liang, C., and Hutchinson, J. W., 1993. Mechanics of the pushout test. Mechanics of Materials, 14, 207-221.

Mackin, T. J., Warren, P. D., and Evans, A. G., 1992. Effects of fiber roughness on interface sliding in composites. Acta Metall. Mater. , 40,1251-7. 
Marshall, D. B., Cox, B. N. and Evans, A. G., 1985. The mechanics of matrix cracking in brittle-matrix fiber composite. Acta Metall., 33, 2013-21.

Marshall, D. B., Shaw, M. C., Morris, W. L., 1992. Measurement of interfacial debonding and sliding resistance in fiber reinforced intermetallics. Acta Metall. Mater., 40, 443-54.

Nairn, J. A., 2000. Exact and variational theorems for fracture mechanics of composites with residual stresses, traction-loaded cracks, and imperfect interfaces. International Journal of Fracture. 105, 243-71.

Outwater, J. O., Murphy, M. C., 1969. On the fracture energy of uni-directional laminates. 24th Annual Technical Conference, 1969, Reinforced Plastic/Composites Division, The Society of the Plastics Industry, Inc., section 11-C, p. 1-8.

Parthasarathy, T. A., Jero, P. D., Kerans, R. D., 1991. Extraction of interface properties from a single fiber push-out test. Scripta Metall. Mater., 25, 2457-62.

Pupurs, A., Varna, J, 2013. Energy release rate based fiber/matrix debond growth in fatigue. Part I: Self-similar crack growth. Mechanics of Advanced Materials and Structures. 20, 276-87.

Ramirez, F. A., Carlsson, L. A., Acha, B. A., 2009. A method to measure fracture toughness of the fiber/matrix interface using the single-fiber fragmentation test. Composites Part A., 40, 679-86.

Sørensen, B. F., Gamstedt, E. K., Østergaard, R. C., Goutianos, S., 2008. Micromechanical model of cross-over fiber bridging - prediction of mixed mode bridging laws. Mechanics of Materials. 40, 220-4.

Sørensen, B. F., and Goutianos, S., 2014. Mixed Mode cohesive law with interface dilatation. Mechanics of Materials. 70, 76-93. 
Tripathi, D., Jones, F. R., 1998. Review: Single fiber fragmentation tests for assessing adhesion in fiber reinforced composites. J. Mater. Sci., 33, 1-16.

Tvergaard, V., Hutchinson, J. W., 1994. Toughness of an interface along a thin ductile layer joining elastic solids. Philosophical Magazine A. 70, 641-56.

Varna, J., Joffe, R., Berglund, L. A., 1996. Interfacial toughness evaluation from the single-fiber fragmentation test. Composites Science and Technology. 56, 1105-9.

Wagner, H. D., and Zhou, X.-F., A twin-fiber fragmentation experiment. Composites part A. 29A, 331-5.

Wagner, H. D., Nairn, J. A., Detassis, M., 1995. Toughness of interfaces from initial fiber-matrix debonding in a single-fiber composite fragmentation test. Applied Composite Materials, 2, 107-117.

Wu, W., Verpoest, I., Varna, J., 2000. Prediction of energy release rate due to the growth of an interface crack by variational analysis. Composites Science and Technology. 60, 351-60. 